\title{
Síntese de pigmentos de níquel utilizando areia descartada de fundição como fonte de sílica
}

\section{(Synthesis of nickel pigments using waste foundry sand as a silica source)}

\author{
A.C.Martin ${ }^{*}$, L. M. Schabbach ${ }^{2}$, M. V. Folgueras ${ }^{1}$ \\ ${ }^{1}$ Universidade do Estado de Santa Catarina, Programa de Pós-graduação em Ciência e \\ Engenharia de Materiais, Joinville, SC, Brasil \\ ${ }^{2}$ Universidade Federal de Santa Catarina, Departamento de Engenharias, Blumenau, SC, Brasil
}

\begin{abstract}
Resumo
Sintetizaram-se pigmentos do sistema $\mathrm{Zn}_{2-\mathrm{x}} \mathrm{Ni}_{\mathrm{x}} \mathrm{SiO}_{4}$ variando a proporção molar de zinco e níquel, partindo da estrutura willemita $\left(\mathrm{Zn}_{2} \mathrm{SiO}_{4}\right)$ à olivina $\left(\mathrm{Ni}_{2} \mathrm{SiO}_{4}\right)$, usando areia descartada de fundição (ADF) e sílica comercial como fontes de $\mathrm{SiO}$. $\mathrm{A} A D F$ foi caracterizada por análise química e DRX. A síntese dos pigmentos seguiu rota cerâmica, com x=0,0,5, 1, 1,5 e 2,0 mol e calcinação a $1200{ }^{\circ} \mathrm{C}$. Os pigmentos foram caracterizados mediante DRX e medidas colorimétricas. Os resultados mostraram que, apesar da presença de alumina e ferro na ADF, as fases cristalinas e cor dos pigmentos foram similares para ambas as fontes de sílica. Teores menores de níquel favoreceram a formação de willemita e maiores a de olivina. Todos os pigmentos (com e sem ADF) dispersos em dois esmaltes e queimados em forno semi-industrial desenvolveram coloração marrom, indicando instabilidade destes nas matrizes usadas, mas confirmando a viabilidade de substituição da sílica comercial por ADF.
\end{abstract}

Palavras-chave: resíduos sólidos, pigmentos cerâmicos, estabilidade cromática.

Abstract

Pigments of $\mathrm{Zn}_{2-x} \mathrm{Ni}_{x} \mathrm{SiO}_{4}$ system were synthesized varying molar ratio of zinc and nickel, starting from willemite $\left(\mathrm{Zn}_{2} \mathrm{SiO}_{4}\right)$ to olivine $\left(\mathrm{Ni}_{2} \mathrm{SiO}_{4}\right)$ structure, using waste foundry sand (WFS) and commercial silica as sources of $\mathrm{SiO}_{2}$. WFS was characterized by chemical analysis and XRD. The synthesis of pigments was performed by ceramic route with $x=0,0.5,1,1.5$ and 2.0 mol and calcination at $1200{ }^{\circ} \mathrm{C}$. They were characterized by XRD and colorimetric measurements. Results showed that, despite the presence of alumina and iron traces in the WFS composition, the crystalline phases of pigments and their colors were similar for both silica sources. Also, lower additions of nickel favored the willemite formation, while higher ones favored the olivine formation. The brown color was noticed when the pigments were dispersed in two types of ceramic glazes and fired in a semi-industrial oven; as a result, instability was identified. However, the feasibility of obtaining pigments by replacing commercial silica with WFS was confirmed. Keywords: solid residues, ceramic pigments, chromatic stability.

\section{INTRODUÇÃO}

O aumento populacional e o consequente crescimento das atividades industriais acarretam em danos à natureza, especialmente devido ao esgotamento de recursos naturais e à geração de resíduos $[1,2]$. Assim, a exploração dos recursos e a problemática relativa ao descarte de resíduos acentuam a necessidade de recuperação destes materiais [3]. Atualmente o procedimento mais adotado é o armazenamento em aterros industriais [4]. No entanto, seu custo e limitação de espaço tornam necessária a busca de alternativas para a utilização destes resíduos [5, 6]. Uma alternativa seria utilizar estes resíduos como matéria-prima em outras atividades [4, 7]. Entende-se que o resíduo de uma empresa pode ser um material com potencial econômico para outra [8], minimizando os problemas ambientais [9]. No âmbito dos resíduos sólidos industriais, têm-se as areias descartadas

*amandacarolinemartin@gmail.com

Dhttps://orcid.org/0000-0003-0362-6757 de fundição (ADF), um dos resíduos com maior volume de geração [10]. A areia utilizada no processo é reutilizada diversas vezes, até ser considerada inadequada e descartada [11]. Estudos foram realizados para avaliar a viabilidade de se utilizar este resíduo como matéria-prima em diferentes produtos, como concreto, argamassa, asfalto, vidros e, em poucos casos, pigmentos cerâmicos [12-20]. A utilização da ADF no campo da construção civil já é bem consolidada, existindo normas específicas de uso [10, 21], contudo seu uso na obtenção de pigmentos cerâmicos ainda é pouco explorado. Em contrapartida, a utilização de diferentes tipos de resíduos para produção de pigmentos vem aumentando [22] devido à busca de matérias-primas alternativas de menor custo [23] e decorrente da preocupação ambiental.

Os pigmentos são substâncias capazes de conferir cor a um meio, no qual são insolúveis e não interagem química nem fisicamente [24]. No setor de revestimentos cerâmicos são importantes materiais para o efeito estético das peças [25], mas podem também ser usados para conferir cor a cosméticos, tintas, cerâmicas em geral, principalmente na 
camada vítrea, e uma variedade de outras aplicações [26]. Os pigmentos podem ser orgânicos ou inorgânicos, coloridos, brancos ou pretos [24]. Os pigmentos inorgânicos possuem estabilidade térmica e química [27-29] e são matériasprimas relevantes na indústria cerâmica [30], sendo um produto de considerável valor agregado. $\mathrm{Na}$ aplicação em esmaltes cerâmicos a coloração das peças é observada após a queima [31] contribuindo para sua função decorativa [32]. A cor desenvolvida pode ser estudada por meio do espaço de cor $\mathrm{L}^{*} \mathrm{a}^{*} \mathrm{~b}^{*}$, que identifica os parâmetros luminosidade ( $\left.\mathrm{L}^{*}\right)$, variação entre verde e vermelho $\left(a^{*}\right)$ e variação entre azul e amarelo (b*) [33-35]. A partir das coordenadas L*a*b* é possível quantificar a diferença de cor entre duas amostras, por meio do valor $\Delta \mathrm{E}^{*}{ }_{\mathrm{ab}}$, obtido por meio da Eq. A [36]. Este valor também pode ser utilizado como indicativo qualitativo da estabilidade e durabilidade do pigmento [34, 37-39].

$$
\Delta \mathrm{E}_{\mathrm{ab}}^{*}=\sqrt{\Delta \mathrm{L}^{2}+\Delta \mathrm{a}^{2}+\Delta \mathrm{b}^{2}}
$$

Dentre os pigmentos inorgânicos, têm-se a willemita $\mathrm{Zn}_{2} \mathrm{SiO}_{4}$ e a olivina de níquel $\mathrm{Ni}_{2} \mathrm{SiO}_{4}$. O silicato de zinco $\left(\mathrm{Zn}_{2} \mathrm{SiO}_{4}\right)$, conhecido por willemita, é de grande interesse devido à sua aplicabilidade industrial [40]. O zinco incorporado na estrutura em forma de óxido não contribui com a cor do pigmento [41,42], porém possibilita a incorporação de metais de transição em posições tetraédricas [43]. A partir da incorporação de uma pequena quantidade de níquel na estrutura da willemita é possível obter pigmentos de cor azul turquesa [22, 44], enquanto a olivina de níquel possui cor verde [45]. No entanto, a interação química e física entre pigmento e esmalte, assim como variações no processo de queima, pode afetar a aparência final da peça cerâmica [46]. Os eventuais óxidos livres do pigmento não são sempre resistentes ao efeito de dissolução em altas temperaturas, podendo ocorrer a degradação da cor inicial do pigmento [47]. Sabe-se que em pigmentos de níquel, a estabilidade do precursor afeta a cinética de reação [44] e que a síntese da olivina de níquel em laboratório é dificultada [48]. Já foi comprovado que a utilização de diferentes resíduos, como ADF precursora de $\mathrm{SiO}_{2}$ e lama de galvanização para incorporação do cromóforo, permitiu a obtenção de olivina $\left(\mathrm{Ni}_{2} \mathrm{SiO}_{4}\right)$ a partir de $1050{ }^{\circ} \mathrm{C}$ [48]. A presença de impurezas nos resíduos pode atuar como mineralizadores ou fundentes, facilitando a síntese do pigmento [23, 49]. Neste trabalho foi utilizada sílica comercial e ADF como precursores de $\mathrm{SiO}_{2}$ para realizar a síntese do pigmento $\mathrm{Z}_{\mathrm{n} 2-\mathrm{x}} \mathrm{Ni}_{\mathrm{x}} \mathrm{SiO}_{4}$, sendo $\mathrm{x}$ a proporção molar dos elementos zinco e níquel ( $\mathrm{x}=0,0,5$, $1,0,1,5$ e $2,0 \mathrm{~mol}$ ). Os pigmentos foram caracterizados por difratometria de raios $\mathrm{X}$ enquanto o efeito pigmentante foi avaliado por colorimetria para avaliar o efeito da substituição da sílica comercial pela areia descartada no processo de síntese e no desenvolvimento da cor.

\section{MATERIAIS E MÉTODOS}

A areia descartada de fundição foi fornecida por uma empresa do setor, localizada em Joinville, SC, região de grande concentração de empresas de fundição e onde a problemática de adequada gestão desse resíduo é relevante. Inicialmente a areia foi calcinada para eliminação de componente orgânico e posteriormente moída por $20 \mathrm{~min}$, em moinho de bolas, para redução do tamanho de partícula e aumento da área superficial e reatividade [50]. A composição química da areia resultante após esses tratamentos foi determinada por espectroscopia de fluorescência de raios X, a partir da média de três análises do mesmo lote. Já a sua estrutura cristalina foi determinada por difração de raios X.

Para fins comparativos, também foi utilizada a sílica comercial, cedida por empresa do setor de pigmentos, com pureza de $96 \%$. Para a obtenção dos pigmentos $\mathrm{Zn}_{2-x} \mathrm{Ni}_{x} \mathrm{SiO}_{4}$ ( $\mathrm{x}=0,0,5,1,1,5$ e $2 \mathrm{~mol}$ ) pela rota cerâmica, o óxido de zinco $(\mathrm{ZnO})$ utilizado foi da Êxodo (pureza 99\%) e óxido de níquel $\left(\mathrm{Ni}_{2} \mathrm{O}_{3}\right)$ da Dinâmica (pureza $99 \%$ ). Os reagentes foram pesados e homogeneizados em meio alcoólico em moinho planetário (Fritsch, Pulverisette 5), por $20 \mathrm{~min}$ a $250 \mathrm{rpm}$. A mistura foi seca sob lâmpada e posteriormente tratada termicamente a $1200{ }^{\circ} \mathrm{C}$, temperatura adequada para obtenção da willemita, com aquecimento de $5{ }^{\circ} \mathrm{C} / \mathrm{min}$ e permanência de $3 \mathrm{~h}$ na temperatura estabelecida $[5,21]$. Após a calcinação as amostras foram desaglomeradas e peneiradas em malha $75 \mu \mathrm{m}$. Na Tabela I são apresentadas as nomenclaturas utilizadas para a identificação dos pigmentos sintetizados em função da composição e fonte de sílica.

Tabela I - Nomenclatura utilizada para identificar os pigmentos obtidos.

[Table I - Codes used to identify the pigments.]

\begin{tabular}{cccc}
\hline $\mathrm{X}$ & $\begin{array}{c}\text { Composto de } \\
\text { interesse }\end{array}$ & $\begin{array}{c}\text { Sílica } \\
\text { comercial }\end{array}$ & Resíduo \\
\hline 0 & $\mathrm{Zn}_{2} \mathrm{SiO}_{4}$ & $\mathrm{Z}_{2} \mathrm{~S}$ & $\mathrm{Z}_{2} \mathrm{R}$ \\
0,5 & $\mathrm{Zn}_{1,5} \mathrm{Ni}_{0,5} \mathrm{SiO}_{4}$ & $\mathrm{~N}_{0,5} \mathrm{~S}$ & $\mathrm{~N}_{0,5} \mathrm{R}$ \\
1,0 & $\mathrm{Zn}_{1} \mathrm{Ni}_{1} \mathrm{SiO}_{4}$ & $\mathrm{~N}_{1} \mathrm{~S}$ & $\mathrm{~N}_{1} \mathrm{R}$ \\
1,5 & $\mathrm{Zn}_{0,5} \mathrm{Ni}_{1,5} \mathrm{SiO}_{4}$ & $\mathrm{~N}_{1,5} \mathrm{~S}$ & $\mathrm{~N}_{1,5} \mathrm{R}$ \\
2,0 & $\mathrm{Ni}_{2} \mathrm{SiO}_{4}$ & $\mathrm{~N}_{2} \mathrm{~S}$ & $\mathrm{~N}_{2} \mathrm{R}$ \\
\hline
\end{tabular}

Os pigmentos foram caracterizados em relação à estrutura cristalina, por meio de difração de raios X (DRX) de $10^{\circ}$ a $70^{\circ}$ (29) com velocidade de $2 \%$ min, e cor, por meio de medida dos parâmetros $\mathrm{L}^{*} \mathrm{a} \mathrm{b}^{*}$, usando um espectrofotômetro Varian Cary 50, considerando o iluminante D65, entre 360 e $830 \mathrm{~nm}$. $\mathrm{O}$ valor $\Delta \mathrm{E}_{\mathrm{ab}}$ foi calculado para avaliar a diferença colorimétrica dos pigmentos sintetizados a partir das duas fontes de sílica. Posteriormente os pigmentos sintetizados obtidos foram dispersos (em percentual mássico de 3\%) em dois esmaltes cerâmicos: um mate/opaco (contendo silicato de zircônio e frita de cálcio e zinco) e um transparente brilhante (contendo sílica, óxido de zinco, óxido de cálcio e alumina), para avaliação da estabilidade de cor. As peças foram submetidas à queima em forno semi-industrial elétrico de uma empresa cerâmica a $1190{ }^{\circ} \mathrm{C}$ com ciclo de queima de 40 min e caracterizadas em espectrofotômetro portátil Ci6X X-Rite com iluminante D65. Também nesse caso, as 
peças obtidas com pigmentos produzidos com resíduo foram comparadas às obtidas com sílica comercial, considerando a variação de cor $\Delta \mathrm{E}_{\mathrm{ab}}$.

\section{RESULTADOS E DISCUSSÃO}

A aparência visual da ADF, como recebida e após tratamento térmico, está apresentada na Fig. 1, onde é evidente a alteração de cor devido ao processo de calcinação, que resultou na decomposição da matéria orgânica presente no resíduo, bem como da amostra após a moagem, devido à redução do tamanho de partícula e maior espalhamento da luz. A composição química dos diferentes lotes de areia descartada de fundição (ADF) analisados após calcinação e moagem é apresentada na Tabela II. Percebeu-se que, mesmo as areias sendo provenientes do mesmo lote, houve pequenas variações na sua composição. Foi possível verificar que o resíduo foi rico em sílica, com teor de aproximadamente $86 \%$, mas também apresentou percentual significativo de óxido de alumínio, na faixa de $9 \%$, além de outros óxidos, como de ferro e titânio. A caracterização por difração de raios $X$ da ADF (Fig. 2) evidenciou a presença de quartzo (JCPD 00-046-1045) como fase cristalina predominante, mas também a presença de picos de baixa intensidade que foram associados à presença de coríndon $\left(\mathrm{Al}_{2} \mathrm{O}_{3}, \mathrm{JCPD} 01\right.$ 071-1127).

Os pigmentos produzidos foram caracterizados em relação à sua estrutura cristalina (Fig. 3) e foi possível observar a semelhança entre os resultados dos pigmentos sintetizados com sílica comercial e com ADF. Com a temperatura de calcinação de $1200^{\circ} \mathrm{C}$ para todas as amostras, evidenciou-se a formação da willemita para os pigmentos com zinco, ainda que com algumas fases residuais de $\mathrm{ZnO}$, para ambas as fontes de sílica, e para os pigmentos obtidos com sílica comercial a fase residual de $\mathrm{SiO}_{2}$. Para a composição com $\mathrm{x}=0,5$ percebeu-se a coexistência das fases

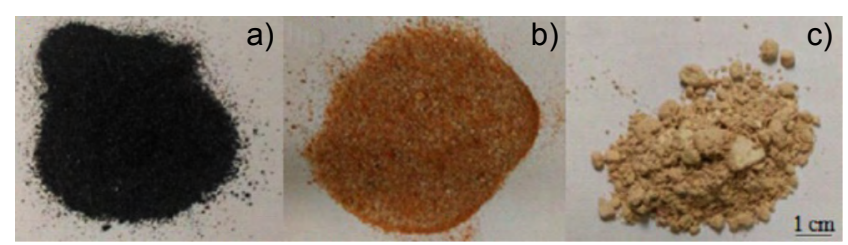

Figura 1: Imagens da ADF: A) como recebida; B) após calcinação; e C) após calcinação e moagem.

[Figure 1: Images of WFS: A) as received; B) after calcination; and $C$ ) after calcination and milling.]

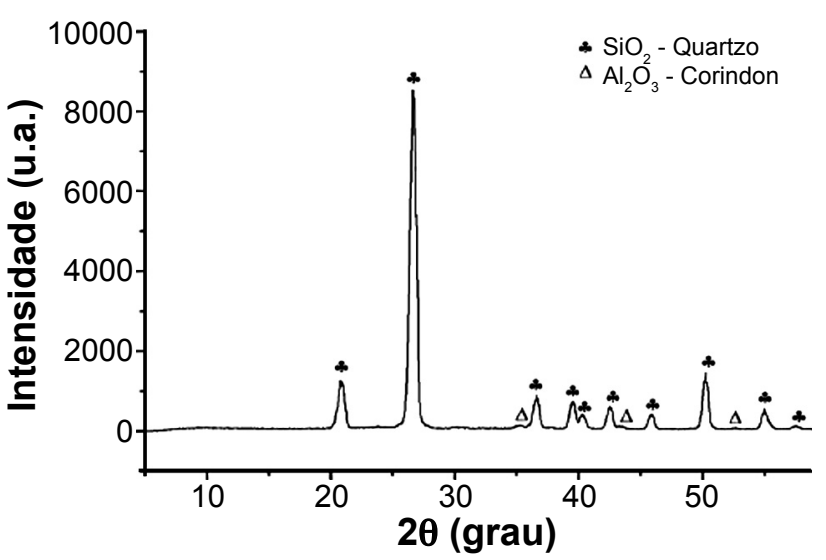

Figura 2: Difratograma de raios $\mathrm{X}$ da $\mathrm{ADF}$ após calcinação e moagem.

[Figure 2: X-ray diffractogram of WFS after calcination and milling.]

de willemita e olivina e, à medida que ocorreu o aumento da quantidade de níquel, observou-se também o incremento na intensidade dos picos representativos da presença de $\mathrm{SiO}_{2}$. Isso indicou que a redução do teor de zinco e aumento do teor de níquel ocasionaram a diminuição da solubilidade entre os componentes envolvidos. Na literatura já se evidenciou em estudos anteriores que para $x=0,3$ há solubilização incompleta de níquel e zinco [22]. Já para $\mathrm{x}=1,5$ na calcinação a $1200{ }^{\circ} \mathrm{C}$, foi verificada a presença de quartzo, níquel e olivina e quando $\mathrm{x}=2$, nesta temperatura, houve a presença discreta de olivina, juntamente com quartzo e níquel [44]. Neste trabalho, quando $\mathrm{x}=2$, não foi possível identificar a formação da fase de interesse $\left(\mathrm{Ni}_{2} \mathrm{SiO}_{4}\right)$, ainda que as impurezas do resíduo pudessem ter atuado como mineralizadores. Em contrapartida, a presença de cristobalita para o pigmento com $\mathrm{ADF}$ em $\mathrm{x}=2$ pode ser indicativa da reatividade distinta entre os precursores de sílica [51]. A uniformidade da distribuição do silício e do níquel no pó resultante da síntese realizada sem o uso de zinco, ou seja $\mathrm{x}=2$, foi confirmada por meio de análise por espectroscopia por dispersão de energia (EDS) acoplada a um microscópio eletrônico de varredura (MEV). A Fig. 4 apresenta as imagens relativas ao mapeamento da presença dos dois elementos no pó tratado termicamente. Assim, a dificuldade em obter o composto, neste caso, pelo método de síntese convencional deve ser explicada apenas pela baixa temperatura utilizada, visto que a temperatura necessária para obtenção de olivina é normalmente superior a $1200{ }^{\circ} \mathrm{C}$ quando realizada a síntese por meio da rota cerâmica [44].

Tabela II - Composição química (\% em massa) da areia descartada de fundição.

[Table II - Chemical composition (wt\%) of waste foundry sand.]

\begin{tabular}{ccccccccccc}
\hline & $\mathrm{SiO}_{2}$ & $\mathrm{Al}_{2} \mathrm{O}_{3}$ & $\mathrm{Fe}_{2} \mathrm{O}_{3}$ & $\mathrm{SO}_{3}$ & $\mathrm{~K}_{2} \mathrm{O}$ & $\mathrm{CaO}$ & $\mathrm{TiO}_{2}$ & Outros & PF & Total \\
\hline ADF1 & 85,95 & 9,35 & 1,11 & 1,38 & 0,79 & 0,43 & 0,38 & 0,31 & 0,30 & 100,00 \\
ADF2 & 85,05 & 9,70 & 1,11 & 1,25 & 0,78 & 0,42 & 0,38 & 1,02 & 0,30 & 100,00 \\
ADF3 & 85,90 & 9,59 & 1,16 & 1,15 & 0,79 & 0,43 & 0,38 & 0,30 & 0,30 & 100,00 \\
Média & 85,63 & 9,55 & 1,13 & 1,26 & 0,78 & 0,43 & 0,38 & 0,54 & 0,30 & 100,00 \\
\hline
\end{tabular}


Em relação ao aspecto visual dos pigmentos, apresentado na Fig. 5, houve variação de cor do branco da willemita, sem
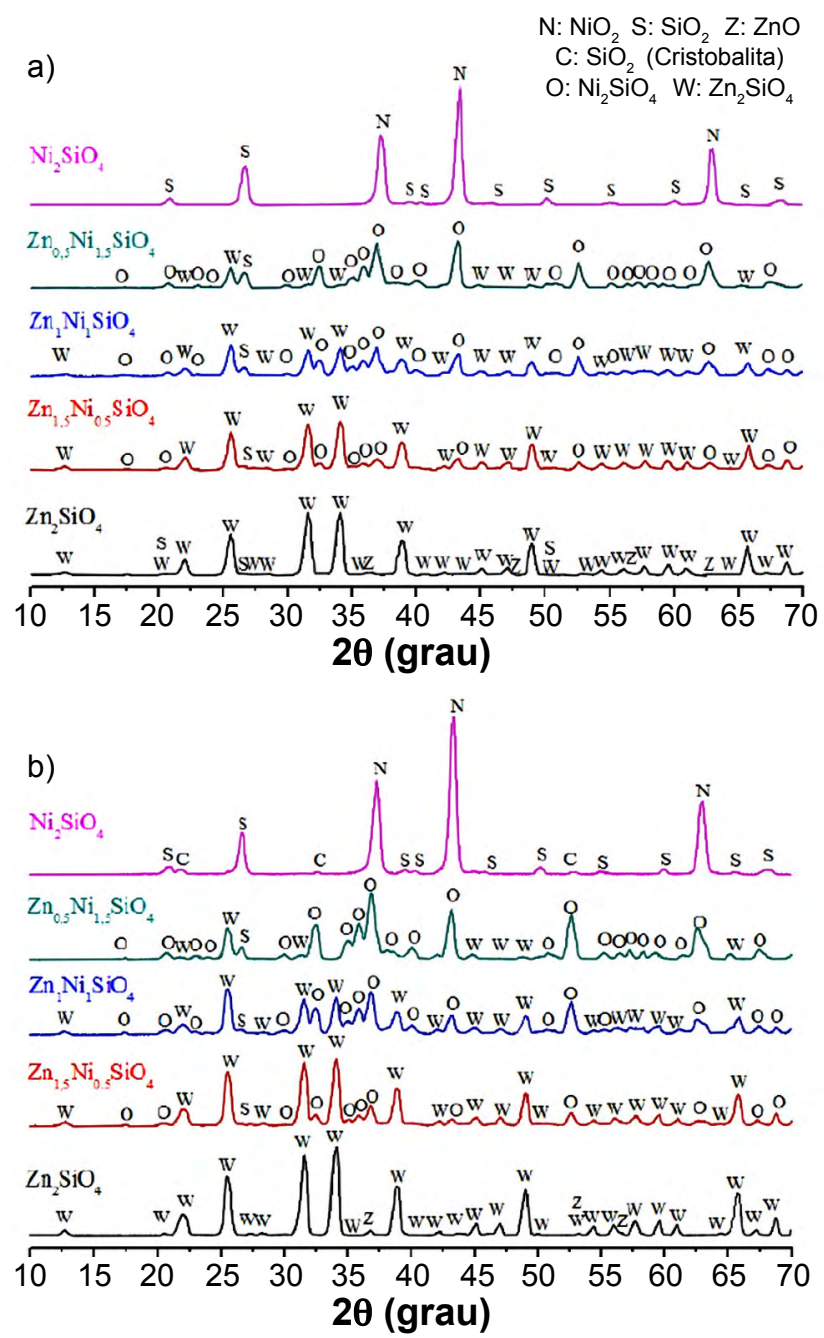

Figura 3: Difratogramas de raios $\mathrm{X}$ das amostras de pigmentos obtidos com sílica comercial (a) e com resíduo (b).

[Figure 3: X-ray diffractograms of pigment samples obtained with commercial silica (a) and with residue (b).]

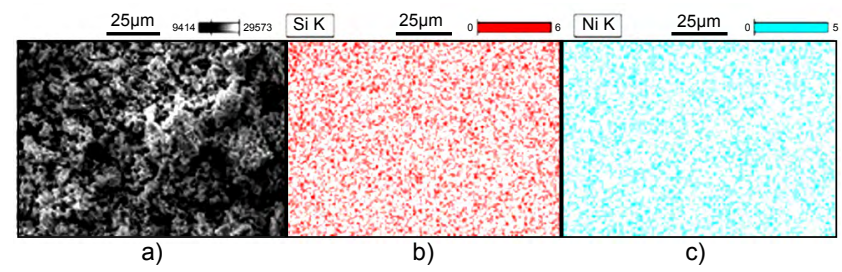

Figura 4: Imagens de MEV (a) e de mapeamento dos elementos $\mathrm{Si}$ (b) e Ni (c) da amostra $\mathrm{N}_{2} \mathrm{~S}$.

[Figure 4: Images of SEM (a) and dot mapping of Si (b) and $\mathrm{Ni}(\mathrm{c})$ elements of the $\mathrm{N}_{2} \mathrm{~S}$ sample.]

cromóforo, ao turquesa da willemita com adição de níquel e, por fim, ao verde ou verde amarelado para a composição sem $\mathrm{ZnO}$. De forma geral, houve semelhança na coloração dos pigmentos obtidos com sílica comercial e resíduo. Apenas houve significativa variação na tonalidade para as amostras com $x=1,5$ e 2,0 em que os pigmentos com resíduo aparentemente foram mais amarelados. Uma melhor caracterização da cor dos pós de pigmentos obtidos foi realizada através da media dos parâmetros colorimétricos ( $\mathrm{L}^{*}$, $a^{*}, b^{*}$ ) do sistema CIELab usando um espectrofotômetro UVvis. As coordenadas $\mathrm{L} * \mathrm{a} * \mathrm{~b} *$ dos pigmentos, apresentados na Tabela III, indicaram que para $\mathrm{x}=0$ e $\mathrm{x}=0,5$ a willemita obtida com resíduo foi significativamente mais clara $(\Delta \mathrm{L}=+15 \mathrm{e}$ +11 , respectivamente) quando comparada à sílica comercial. Já para as amostras que consideraram maiores adições de cromóforo, os valores negativos de $\mathrm{a}^{*}$ foram indicativos da cor verde e, com exceção da amostra $\mathrm{N}_{0,5} \mathrm{~S}$, os valores

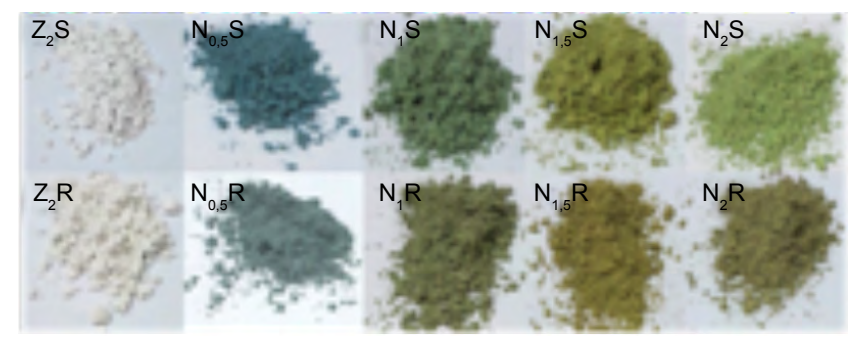

Figura 5: Imagens mostrando a cor dos pigmentos. [Figure 5: Images showing the color of pigments.]

Tabela III - Parâmetros $\mathrm{L}^{*} \mathrm{a}{ }^{*} \mathrm{~b}$ dos pigmentos na forma de pó.

[Table III $-L^{*} a * b^{*}$ parameters of pigments.]

\begin{tabular}{ccccccc}
\hline Amostra & $\mathrm{L}^{*}$ & $\mathrm{a}^{*}$ & $\mathrm{~b}^{*}$ & $\Delta \mathrm{L}$ & $\Delta \mathrm{a}$ & $\Delta \mathrm{b}$ \\
\hline $\mathrm{Z}_{2} \mathrm{~S}$ & 26,33 & 0,20 & $-0,73$ & 15,47 & $-0,32$ & 0,26 \\
$\mathrm{Z}_{2} \mathrm{R}$ & 41,80 & $-0,12$ & $-0,47$ & & & \\
$\mathrm{~N}_{0,5} \mathrm{~S}$ & 68,94 & $-14,63$ & $-5,32$ & 11,52 & 5,12 & 29,22 \\
$\mathrm{~N}_{0,5} \mathrm{R}$ & 80,46 & $-9,51$ & 23,90 & & & \\
$\mathrm{~N}_{1} \mathrm{~S}$ & 79,31 & $-16,55$ & 16,57 & & & \\
$\mathrm{~N}_{1} \mathrm{R}$ & 72,09 & $-10,11$ & 16,86 & $-7,22$ & 6,44 & 0,29 \\
$\mathrm{~N}_{1,5} \mathrm{~S}$ & 74,15 & $-12,14$ & 28,98 & & & \\
$\mathrm{~N}_{1,5} \mathrm{R}$ & 78,43 & $-6,038$ & 30,92 & 4,28 & 6,10 & 1,94 \\
$\mathrm{~N}_{2} \mathrm{~S}$ & 89,79 & $-17,15$ & 24,08 & & & \\
$\mathrm{~N}_{2} \mathrm{R}$ & 80,46 & $-9,511$ & 23,90 & $-9,33$ & 7,64 & $-0,18$ \\
\hline
\end{tabular}


positivos de $\mathrm{b}^{*}$ representaram a cor amarelada. $\mathrm{O}$ valor $\Delta \mathrm{a}$ positivo foi indicativo da coloração menos esverdeada das amostras com resíduo e o valor positivo de $\Delta \mathrm{b}$ apontou a cor mais amarelada das amostras com resíduo, com exceção do pigmento $\mathrm{N}_{2} \mathrm{R}$, em que essa variação, por ser muito pequena, pode ser considerada nula. Observou-se, portanto, que as coordenadas $\mathrm{L}^{*} \mathrm{a} \mathrm{b}^{*}$ e suas respectivas diferenças $(\Delta)$ foram coerentes com o aspecto visual identificado, mostrando que os pigmentos sintetizados com sílica comercial apresentaram coloração mais esverdeada. Ressalta-se que não é somente relevante a cor observada no pigmento na forma de pó; sua estabilidade térmica e química no meio ao qual é disperso é de fundamental importância [29,52].

Os pigmentos foram aplicados em esmalte mate/opaco e transparente brilhante, como apresentado na Fig. 6. Em ambos, observou-se que a cor desenvolvida na matriz vitrea usada foi significamente distinta da cor dos pigmentos na forma de pó, evidenciando a instabilidade química desses pigmentos nessas matrizes, que pode estar associada à presença de fases resíduais presentes nas amostras (como mostra os difratogramas de raios X da Fig. 3) [47]. A partir da Fig. 6a é possível observar que a intensidade de cor aumentou à medida que se aumentou a concentração de cromóforo adicionado. Ressalta-se que para para ambos os tipos de pigmentos (com ADF e sílica comercial) a coloração desenvolvida foi similar tendendo ao marrom. A Tabela IV apresenta os valores das coordenadas cromáticas do sistema CIELab dos esmaltes opacos pigmentados, bem como a diferença de cor desenvolvida por cada pigmento quando se comparou os vidrados com pigmentos ADF e sílica comercial. Notou-se que para os pigmentos com proporção molar de níquel inferior ou igual a 1,5 a diferença de cor foi menor ou igual a 1 , podendo ser considerada insignificante, o que mostrou a similaridade do desenvolvimento da cor dos pigmentos sintetizados com ambas as fontes de sílica (comercial e resíduo). Já para a proporção molar de níquel $\mathrm{x}=2,0$ observou-se uma diferença de cor significativa $(\Delta \mathrm{E}=2,80)$ com maior evidência de variação na luminosidade
(L*) e no parâmetro $b^{*}$, confirmando o que se observou na percepção visual, ou seja, o pigmento com $x=2,0$ com ADF desenvolveu uma coloração significativamente mais escura $(\Delta \mathrm{L}=-2,70)$ e levemente menos amarelada $(\Delta \mathrm{b}=-0,77)$ nessa matriz vítrea do que o pigmento com sílica comercial com a mesma composição de níquel.

$\mathrm{Na}$ Fig. 6b é apresentado o aspecto visual das amostras de vidrado transparente brilhante pigmentadas com os pigmentos obtidos com sílica comercial e ADF. Nesse caso, também foi possível observar grande semelhança de tonalidade desenvolvida com as amostras obtidas com os pigmentos sintetizados com ambas as fontes de sílica. $\mathrm{Na}$ Tabela V são apresentados os parâmetros colorimétricos $\mathrm{L} * \mathrm{a} * \mathrm{~b} *$ do sistema CIELab dos vidrados transparentes com $3 \%$ em massa dos pigmentos sintetizados. Observou-se que novamente a maior diferença de cor ocorreu com as amostras contendo maior percentual de cromóforo $(x=2,0)$. No entanto,

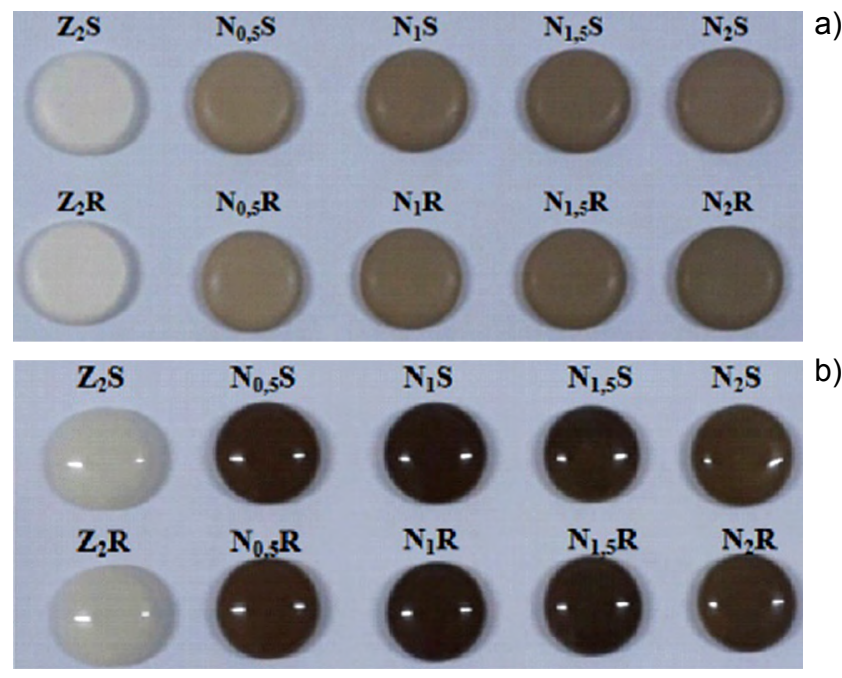

Figura 6: Amostras de esmaltes mate/opaco (a) e transparente brilhante (b) com $3 \mathrm{wt} \%$ dos pigmentos sintetizados.

[Figure 6: Samples of matt opaque (a) and bright transparent (b) enamels with $3 \mathrm{wt} \%$ of synthesized pigments.]

Tabela IV - Parâmetros L*a*b* para esmalte mate/opaco com $3 \mathrm{wt} \%$ dos pigmentos sintetizados. [Table IV - Parameters $L * a * b *$ for matt/opaque enamel with $3 \mathrm{wt} \%$ of synthesized pigments.]

\begin{tabular}{cccccccc}
\hline Amostra & $\mathrm{L}^{*}$ & $\mathrm{a}^{*}$ & $\mathrm{~b}^{*}$ & $\Delta \mathrm{L}$ & $\Delta \mathrm{a}$ & $\Delta \mathrm{b}$ & $\Delta \mathrm{E}$ \\
\hline $\mathrm{Z}_{2} \mathrm{~S}$ & 91,03 & $-0,68$ & 7,18 & & & & \\
$\mathrm{Z}_{2} \mathrm{R}$ & 91,48 & $-0,66$ & 7,36 & 0,45 & 0,02 & 0,18 & 0,49 \\
$\mathrm{~N}_{0,5} \mathrm{~S}$ & 71,78 & 3,20 & 15,26 & & & & \\
$\mathrm{~N}_{0,5} \mathrm{R}$ & 72,28 & 3,04 & 15,09 & 0,50 & $-0,16$ & $-0,17$ & 0,55 \\
$\mathrm{~N}_{1} \mathrm{~S}$ & 66,64 & 3,81 & 15,72 & & & & \\
$\mathrm{~N}_{1} \mathrm{R}$ & 66,64 & 3,76 & 16,23 & 0,00 & $-0,05$ & 0,51 & 0,51 \\
$\mathrm{~N}_{1,5} \mathrm{~S}$ & 63,90 & 3,89 & 15,59 & & & & \\
$\mathrm{~N}_{1,5} \mathrm{R}$ & 63,74 & 3,82 & 16,69 & $-0,16$ & $-0,07$ & 1,10 & 1,11 \\
$\mathrm{~N}_{2} \mathrm{~S}$ & 65,08 & 3,94 & 16,58 & & & & \\
$\mathrm{~N}_{2} \mathrm{R}$ & 62,38 & 3,17 & 16,47 & $-2,70$ & $-0,77$ & $-0,11$ & 2,81 \\
\hline
\end{tabular}


Tabela V - Parâmetros L*a*b* para o esmalte transparente brilhante com $3 \mathrm{wt} \%$ dos pigmentos sintetizados. [Table $V$ - Parameters $L * a * b *$ for bright transparent enamel with $3 \%$ of synthesized pigments.]

\begin{tabular}{cccccccc}
\hline Amostra & $\mathrm{L}^{*}$ & $\mathrm{a}^{*}$ & $\mathrm{~b}^{*}$ & $\Delta \mathrm{L}$ & $\Delta \mathrm{a}$ & $\Delta \mathrm{b}$ & $\Delta \mathrm{E}$ \\
\hline $\mathrm{Z}_{2} \mathrm{~S}$ & 84,21 & $-1,58$ & 9,18 & & & & \\
$\mathrm{Z}_{2} \mathrm{R}$ & 83,24 & $-1,47$ & 9,43 & $-0,97$ & 0,11 & 0,25 & 1,01 \\
$\mathrm{~N}_{0,5} \mathrm{~S}$ & 44,56 & 7,05 & 16,32 & & & & \\
$\mathrm{~N}_{0,5} \mathrm{R}$ & 47,22 & 6,60 & 13,67 & 2,66 & $-0,45$ & $-2,65$ & 3,78 \\
$\mathrm{~N}_{1} \mathrm{~S}$ & 40,82 & 6,60 & 13,67 & & & & \\
$\mathrm{~N}_{1} \mathrm{R}$ & 41,51 & 6,58 & 13,92 & 0,69 & $-0,02$ & 0,25 & 0,73 \\
$\mathrm{~N}_{1,5} \mathrm{~S}$ & 44,41 & 5,44 & 14,84 & $-1,48$ & 0,23 & $-1,17$ & 1,90 \\
$\mathrm{~N}_{1,5} \mathrm{R}$ & 42,93 & 5,67 & 13,67 & & & & \\
$\mathrm{~N}_{2} \mathrm{~S}$ & 51,90 & 4,33 & 17,79 & $-3,74$ & 0,39 & $-1,18$ & 3,94 \\
$\mathrm{~N}_{2} \mathrm{R}$ & 48,16 & 4,72 & 16,61 & & & & \\
\hline
\end{tabular}

é importante destacar que os vidrados para as amostras de pigmento com $\mathrm{x}=2$ obtidos com resíduo apresentaram valores de luminosidade $\left(\mathrm{L}^{*}\right)$ significativamente menores. Esse aspecto é positivo para o desenvolvimento do pigmento, pois indica que com uma adição percentual menor deste já seria possível desenvolver cor similar. Ou seja, pigmentos com alto valor de luminosidade (claros) em geral podem apresentar menor poder pigmentante. Para essas amostras de esmaltes transparentes, foi possível observar que para $\mathrm{x}=0,5$ também houve diferença de cor significativa $(\Delta \mathrm{E}=3,78)$, com distribuição dessa diferença nos parâmetros $L^{*} \mathrm{e} \mathrm{b*}$, ou seja, a amostra com o pigmento ADF se mostrou mais clara e menos amarela. Embora essa variação se mostrou significativa, na observação visual (Fig. 6b) ela foi somente pouco perceptível, tanto para essa amostra quanto para amostra com $x=2,0$.

Outros autores também identificaram a instabilidade de cor de diferentes pigmentos de níquel quando aplicados em esmaltes cerâmicos, confirmando a formação da cor marrom ou, em alguns casos, amarelo-esverdeado [22, 44, 47]. A ocorrência deste fenômeno pode ser justificada pela possível presença de óxido livre de níquel [48], não resistente à dissolução em esmaltes à alta temperatura, degradando a cor inicial do pigmento [47]. Portanto, embora a ADF apresente impurezas, como coríndon e óxidos de ferro e titânio, além da sílica, foi possível verificar a viabilidade de substituir a sílica comercial por este resíduo para a síntese dos pigmentos, visto que as estruturas cristalinas e desenvolvimento de cor em diferentes esmaltes foram similares para ambas as fontes de $\mathrm{SiO}_{2}$.

\section{CONCLUSÕES}

Os pigmentos obtidos com sílica comerciale como resíduo ADF apresentaram estrutura cristalina e desenvolvimento de cor semelhantes, indicando que a presença de impurezas como coríndon e óxidos de ferro e titânio não interferiram significativamente no processo de síntese. Já em relação à cor as diferenças mais significativas foram observadas para os pigmentos obtidos com maiores teores de níquel $\left(\mathrm{NiO}_{2}\right)$, ou seja $\mathrm{x}=2,0$, tanto para os pigmentos na forma de pó quanto dispersos nos vidrados mate/opaco e transparente. Houve significativa variação de cor quando comparados os pigmentos na forma de pó e após a dispersão em esmaltes cerâmicos, variando do azul-turquesa ou verde para marrom, indicando a instabilidade dos pigmentos. Essa instabilidade foi provavelmente devida à presença de fases cristalinas residuais da síntese, presentes em todos os tipos de pigmentos, e também à composição química dos esmaltes utilizados. A cor desenvolvida nos vidrados, apesar de diferir dos pigmentos na forma de pó, pode ser de interesse comercial. Assim, com os resultados obtidos, foi possível validar a substituição da sílica comercial pelo resíduo ADF na obtenção dos pigmentos, pois as estruturas cristalinas obtidas e a coloração desenvolvida para ambas as fontes de sílica foram similares.

\section{AGRADECIMENTOS}

As autoras são gratas à CAPES e FAPESC pelo apoio financeiro, à empresa Portobello, por gentilmente ceder os esmaltes e realizar as queimas e medidas colorimétricas das peças, e também à empresa fornecedora do resíduo. $\mathrm{O}$ presente trabalho foi realizado com apoio da Coordenação de Aperfeiçoamento de Pessoal de Nível Superior - Brasil (CAPES) - Código de Financiamento 001.

\section{REFERÊNCIAS}

[1] S.S. Rath, D.S. Rao, S.K. Tripathy, S.K. Biswal, Process Saf. Environ. Prot. 117 (2018) 232.

[2] N.P. Stochero, E. Marangon, A.S. Nunes, M.D. Tier, Ceram. Int. 43 (2017) 13875.

[3] J. Carneiro, D.M. Tobaldi, M.N. Capela, R.M. Novais, M.P. Seabra, J.A. Labrincha, Waste Manag. 80 (2018) 371.

[4] J. Carneiro, M.N. Capela, D.M. Tobaldi, R.M. Novais, M.P. Seabra, J.A. Labrincha, Mater. Lett. 223 (2018) 166.

[5] O. Yazoghli-Marzouk, N. Vulcano-Greullet, L. Cantegrit, 
L. Friteyre, A. Jullien, Constr. Build. Mater. 61 (2014) 69.

[6] R. Siddique, Procedia Eng. 95 (2014) 335.

[7] C. Gargori, S.R. Prim, M. Lusar, M.V. Folgueras, G. Monrós, Mater. Lett. 218 (2018) 341.

[8] H. Ovčačíková, J. Vlček, M. Klárová, M. Topinková, Ceram. Int. 43 (2017) 7789.

[9] V. Mymrin, K. Alekseev, R.E. Catai, A. Nagalli, Y.K. Aibuldinov, N.S. Bekturganov, J.L. Rose, R.L.S. Izzo, J. Environ. Chem. Eng. 4 (2016) 753.

[10] R.L.P. Carnin, C. Silva, R. Pozzi, D. Cardoso, Rev. Paviment V (2010) 56.

[11] A. Torres, L. Bartlett, C. Pilgrim, Constr. Build. Mater. 135 (2017) 674.

[12] Y. Aggarwal, R. Siddique, Constr. Build. Mater. 54 (2014) 210 .

[13] A.C. Martin, O.K. Ueno, M.V. Folgueras, in Anais $22^{\circ}$ Congr. Bras. Eng Ciên. Mater., Natal (2016).

[14] R. Siddique, G. Singh, R. Belarbi, K. Ait-Mokhtar, Kunal, Constr. Build. Mater. 83 (2015) 216.

[15] R. Siddique, G. Singh, M. Singh, J. Clean. Prod. 172 (2018) 1111.

[16] J.M.L. Reis, R. Travincas, L.M. Paim, Theor. Appl. Fract. Mech. 56 (2011) 122.

[17] R. Alonso-Santurde, A. Andrés, J.R. Viguri, M. Raimondo, G. Guarini, C. Zanelli, M. Dondi, J. Environ. Manage. 92 (2011) 994.

[18] J.K. Nyembwe, M.E. Makhatha, F.C. Banganayi, K. Nyembwe, Waste Biomass Valori. 9 (2018) 1681.

[19] P.P.O.L. Dyer, M.G. de Lima, L.M.G. Klinsky, S.A. Silva, G.J.L. Coppio, Constr. Build. Mater. 171 (2018) 474. [20] T. Manoharan, D. Laksmanan, K. Mylsamy, P. Sivakumar, A. Sircar, Waste Manag. 71 (2018) 454.

[21] NBR 15702, "Areia descartada de fundição: diretrizes para aplicação em asfalto e em aterro sanitário", Ass. Bras. Normas Técn. (2009).

[22] L.A. Vieira, M.V. Folgueras, M. Tomiyama, S.R. Prim, Mater. Sci. Forum 912 (2018) 44.

[23] G. Costa, M.J. Ribeiro, J.A. Labrincha, M. Dondi, F. Matteucci, G. Cruciani, Dyes Pigm. 78 (2008) 157.

[24] A. Gürses, M. Açıkyıldız, K. Güneş, M.S. Gürses, Dyes and pigments, Springer (2016).

[25] N.M. Ahmed, W.M.A. El-Gawad, M.G. Mohamed, A.A. Elshami, Prog. Org. Coat. 101 (2016) 309.

[26] G. Buvaneswari, V. Aswathy, R. Rajakumari, Dyes Pigm. 123 (2015) 413.

[27] K. Kusumoto, J. Ceram. Soc. Japan 126 (2018) 408.

[28] L.M. Schabbach, F. Bondioli, M.C. Fredel, Dyes Pigm. 99 (2013) 1029.

[29] L.M. Schabbach, F. Bondioli, A.M. Ferrari, T. Manfredini, C.O. Petter, M.C. Fredel, J. Eur. Ceram. Soc. 27 (2007) 179.
[30] W. Hajjaji, M.P. Seabra, J.A. Labrincha, J. Hazard. Mater. 185 (2011) 619.

[31] L. Verger, O. Dargaud, N. Menguy, D. Troadec, L. Cormier, J. Non. Cryst. Solids 459 (2017) 184.

[32] M. Gajek, J. Partyka, M. Lésniak, A. Rapacz-Kmita, L. Wójcik, Ceram. Int. 44 (2018) 15845.

[33] A. Zhang, B. Mu, Z. Luo, A. Wang, Dyes Pigm. 139 (2017) 473.

[34] B. Bae, S. Tamura, N. Imanaka, Ceram. Int. 43 (2017) 7366.

[35] Y.F. Gomes, J. Li, K.F. Silva, A.A.G. Santiago, M.R.D. Bomio, C.A. Paskocimas, M.A. Subramanian, F.V. Motta, Ceram. Int. 44 (2018) 11932.

[36] H.R. Hedayati, A.A.S. Alvani, H. Sameie, R. Salimi, S. Moosakhani, F. Tabatabaee, A.A. Zarandi, Dyes Pigm. 113 (2015) 588

[37] S. Jose, A. Prakash, S. Laha, S. Natarajan, M.L. Reddy, Dyes Pigm. 107 (2014) 118.

[38] H. Erznožnik, T. Razboršek, M.K. Gunde, Prog. Org. Coat. 99 (2016) 47.

[39] S. Zhang, Z. Pan, Y. Wang, Particuology 41 (2018) 20. [40] N.M. Rasdi, Y.W. Fen, N.A.S. Omar, R.S.Azis, M.H.M. Zaid, Results Phys. 7 (2017) 3820.

[41] A.E. Lavat, G.X. Gayo, Ceram. Int. 40 (2014) 11947.

[42] B. Karasu, S. Turan, J. Eur. Ceram. Soc. 22 (2002) 1447.

[43] R.M. Krsmanović, Ž. Antić, M. Mitrić, M.D. Dramićanin, M.G. Brik, Appl. Phys. A Mater. Sci. Process. 104 (2011) 483.

[44] A. Forés, M. Llusar, J.A. Badanes, J. Calbo, M.A. Tena, G. Monrós, Am. Ceram. Soc. Bull. 80 (2001) 47.

[45] M. Llusar, T. Bermejo, J.E. Primo, C. Gargori, V. Esteve, G. Monrós, Ceram. Int. 43 (2017) 9133.

[46] F. Bondioli, T. Manfredini, M. Romagnoli, J. Am. Ceram. Soc. 26 (2006) 311.

[47] M. El Hadri, H. Ahamdane, M.A.I. Raghni, J. Eur. Ceram. Soc. 35 (2015) 765.

[48] D. Esteves, W. Hajjaji, M.P. Seabra, J.A. Labrincha, J. Eur. Ceram. Soc. 30 (2010) 3079.

[49] W. Hajjaji, M.P. Seabra, J.A. Labrincha, Dyes Pigm. 83 (2009) 385.

[50] P.W. Atkins, T.L. Overton, J.P. Rourke, M.T. Weller, F.A. Armstrong, Shriver and Atkins' inorganic chemistry, W.H. Freeman (2010).

[51] F. Andreola, L. Barbieri, F. Bondioli, Dyes Pigm. 94 (2012) 207.

[52] G. Monrós, A. García, J.A. Badenes, M.A. Tena, El color de la cerámica: nuevos mecanismos en pigmentos para los nuevos procesados de la industria cerámica, Athenea (2003).

(Rec. 22/03/2019, Rev. 08/05/2019, Ac. 14/05/2019) 